\title{
Safety of rituximab in the routine treatment of rheumatoid arthritis in Italy in patients refractory to anti-TNF $\alpha$ drugs: results from the observational retrospective-prospective RUBINO study
}

\author{
L. Bazzichi', D. Biasi², E. Tinazzi ${ }^{2}$, M. Muratore ${ }^{3}$, R. Pellerito ${ }^{4}$, R. Russo ${ }^{5}$, \\ M. Corsaro Santi ${ }^{6}$, P. De Sandre ${ }^{7}$, O. Epis ${ }^{8}$, M. Granata ${ }^{9}$, B. Kroegler ${ }^{10}$, \\ C. Meschini ${ }^{11}$, F. Versace ${ }^{12}$, C. Astolfi ${ }^{13}$ on behalf of the RUBINO study group \\ ${ }^{1} \mathrm{AOU}$ Pisana Ospedale Santa Chiara, Pisa; ${ }^{2} \mathrm{AOU}$ Integrata, Verona; ${ }^{3}$ Ospedale Galateo, Lecce; \\ ${ }^{4}$ A.S. O. Ordine Mauriziano di Torino, Torino; ${ }^{5}$ AORN A. Cardarelli, Napoli; \\ ${ }^{6}$ Ospedale San Giovanni Bosco, Napoli; ${ }^{7}$ Ospedale San Bortolo, Vicenza; \\ ${ }^{8}$ Ospedale Niguarda Ca' Granda, Milano; ' $A$ AO San Filippo Neri, Roma; ${ }^{10}$ Policlinico Tor Vergata, Roma; \\ ${ }^{11}$ Ospedale Belcolle, Viterbo; ${ }^{12}$ Ospedale San Paolo, Savona; ${ }^{13}$ Roche Italia S.p.A, Monza, Italy
}

\section{SUMMARY}

The paper reports the results from the observational retrospective-prospective RUBINO study conducted in Italy to assess the safety of rituximab in the treatment of rheumatoid arthritis (RA) in routine clinical practice. The percentage of patients who manifested at least one grade 3 or 4 adverse event (AE) assessed by the Common Terminology Criteria for Adverse Events version 3.0 (CTCAE v.3) during the observation period (primary objective) was evaluated. The percentage of patients manifesting a severe AE (SAE), clinical response to rituximab treatment, clinical remission according to disease activity score for 28 joints (DAS28) criteria, markers of disease and quality of life were also assessed.

Fifty-three Italian rheumatology centers took part in the study. Patients with a diagnosis of RA and inadequate response to anti-tumor necrosis factor $\beta$ (anti-TNF $\alpha$ ) drugs were enrolled. Participating patients had previously received at least one cycle of rituximab, and treatment was still ongoing at the time of recruitment.

Out of 205 patients enrolled, $60 \%$ manifested no form of AE, 14.2\% had at least one grade 3 or 4 AE, and $11.2 \%$ patients reported an SAE.

The overall percentage of patients manifesting AEs (40\%) was lower compared to the DANCER (81\% and 85\%), REFLEX (85\%) and RESET (85\% and 69\%) studies, but higher than that observed in the CERERRA registry (from $10.2 \%$ to $13.9 \%$ ). This difference may be due to the shorter observation period applied in the CERERRA registry (only 12 months) compared to the RUBINO study (up to 3 years). All parameters of RA activity (erythrocyte sedimentation rate, C-reactive protein, health assessment questionnaire score, DAS28) improved significantly during the study.

Key words: Rituximab, Rheumatoid arthritis Anti-TNF failures, Safety.

Reumatismo, 2014; 66 (3): 224-232

\section{INTRODUCTION}

heumatoid arthritis (RA) is a chronic inflammatory and immune-mediated systemic pathological condition with a multifactorial etiology. Progression of joint damage, physical disability and reduced quality of life (QOL) are the essential aspects of this disease (1-5).
In clinical practice, approximately $30 \%$ of RA patients interrupt treatment with antiTNF drugs during the first year due to a lack of efficacy or onset of adverse events (6-9). The clinical efficacy of rituximab in terms of response to the American College of Rheumatology (ACR) and of the European League Against Rheumatism (EULAR) criteria was investigated in two controlled, 
double blind clinical trials (DANCER and REFLEX) with good results. In the DANCER study, $73 \%$ and $67 \%$ of patients receiving $2 \times 1000 \mathrm{mg}$ and $2 \times 500 \mathrm{mg}$ of rituximab respectively achieved a moderate to good response at week 24. In the REFLEX study, a $51 \%$ increase in ACR 20 and a $65 \%$ response to EULAR were observed $(10,11)$. In patients treated with rituximab a significant reduction in disease activity and health assessment questionnaire (HAQ) disability index were observed compared to patients treated with methotrexate single therapy. The studies cited above provide further confirmation of the good safety profile of rituximab. The percentage of patients on rituximab reporting adverse events ranges from $81 \%$ to $85 \%$, compared with $70 \%$ of patients receiving placebo. The most common adverse events were infusion reactions of mild to moderate intensity, which were more frequent during the first infusion and decreased following administration of glucocorticoids. In the REFLEX study, only $18 \%$ of patients treated with rituximab plus methotrexate reported severe grade (3 and 4) adverse events. This percentage does not appear to differ significantly from that reported by patients receiving placebo plus methotrexate. Serious adverse events (SAE) were reported by $7 \%$ of patients in the rituximab plus methotrexate group, and by $10 \%$ in the placebo plus methotrexate group. The safety profile of rituximab was also investigated in long-term studies showing that the repeated depletion of B lymphocytes is well tolerated over time (12). One study reported how, in patients failing to respond to TNF $\alpha$ inhibitors, the safety profile remained unchanged after repeated courses of rituximab. Likewise this trend remained constant in patients treated with up to seven cycles at 6-12 month intervals $(13,14)$.

The economic impact of RA and its consequences on QOL acquire an increasingly important role with worsening of the disease and disease duration (15). Recent studies have indicated that biological medicines such as rituximab are characterized by more favorable cost/efficacy and cost/ usefulness ratios compared to traditional treatments, thus playing a central role in the treatment of RA (16-20). The aim of the RUBINO study was to gather information from routine clinical practice on the use of rituximab and its related clinical impacts, with specific focus on the incidence of adverse events (AE/SAE). The primary endpoint was to evaluate the percentage of patients manifesting at least one grade 3 or 4 adverse event [according to Common Terminology Criteria for Adverse Events version 3.0 (CTCAE v.3)] during the observation period. Furthermore, the study evaluated: the percentage of patients who i) manifested at least one SAE, ii) interrupted rituximab treatment, iii) underwent a second treatment cycle, as well as time to retreatment. Time to response and identification of factors affecting response to rituximab were also assessed. Finally, the study aimed to evaluate clinical remission according to disease activity score (DAS28) criteria, and correlation with patients' QOL during rituximab treatment.

\section{MATERIALS AND METHODS}

An observational, retrospective and prospective multi-center cohort study was carried out. Fifty-three Italian rheumatology centers took part in the study. Eligible subjects were adult male and female patients with a diagnosis of RA according to the ACR 1987 revised criteria (21), who had displayed inadequate response or intolerance to TNF $\alpha$ inhibitors and had started treatment with rituximab over the 12 months prior to the study and were still on treatment at the time of recruitment. Patients with severe active infections, hypersensitivity to the active component or to murine proteins of rituximab, degree IV congestive heart failure, pregnancy or concomitant participation in other clinical trials were not treated with rituximab.

All eligible patients gave their written informed consent to take part in the study. As this was an observational trial, standard treatments were prescribed and no additional diagnostic or monitoring procedures were applied. Subjects were followed pro- 
spectively for 2 years following recruitment. Assessments were undertaken at enrolment and after 6, 12 and 24 months. Information relating to patients' socio-demographic characteristics, concomitant diseases and history of RA were collected at the time of enrolment. If available, the results of laboratory tests [erythrocyte sedimentation rate (ESR), C-reactive protein (CRP), rheumatoid factor (RF), anti-cyclic citrullinated peptide antibodies (anti-CCP)] and DAS28 were also gathered from the beginning of rituximab treatment until completion of the observation period. DAS28 total score was calculated using ESR or CRP according to site practice, together with the presence of concurrent medical conditions and exam availability.

The severity of AE was assessed using the CTCAE 2006 (22). QOL was evaluated using HAQ at each visit (23-25).

Concomitant pharmacological RA treatments were noted.

A list of all adverse events manifested from the start of rituximab treatment until the end of the observation period was compiled. An AE was defined as onset of any sign, symptom, unwanted or unexpected clinical condition after the start of the treatment. A SAE was defined as any clinical event, not necessarily correlated to the administration of the drug, which resulted in death, was life-threatening, required hospitalization or prolongation of hospitalization, resulted in persistent/significant disability/incapacity, was associated with a congenital anomaly/ birth defect or required intervention. $\mathrm{AE}$ description, start/end date, severity classification criteria, intensity, outcome, suspected correlation with the drug, and measures implemented were recorded.

Time to retreatment with rituximab was evaluated by means of Kaplan Meier analysis. Response to rituximab was measured as the first assessment of a $\triangle \mathrm{DAS} 28$ score $>1.2$. $\triangle$ DAS28 identified variation between DAS28 scores obtained at the beginning of rituximab treatment and those detected at subsequent visits. The following predictive factors of response to rituximab were recorded at the start of treatment: gender, age, number of concomitant diseases, RF,
anti-CCP antibodies, DAS28 score, previous number of anti-TNF $\alpha$ drugs administered.

\section{Statistical analysis}

Mean, standard deviation (SD), median, interquartile range, minimum, and maximum values were used for continuous variables, and absolute and relative frequency distributions for categorical variables. Kaplan Meier analysis was also performed on time to response, defined as the time (in days) from the start of observation to the first assessment of a $\triangle D A S 28>1.2$. The percentage of patients in remission was calculated as the number of patients with DAS $28<2.6$ at 6,12 and 24 months. QOL was measured and the score obtained related to achievement of remission. Use of health resources during the observation period was analyzed in terms of number of visits to the general practitioner or specialist consultants, as well as number, type and duration of hospitalization for RA.

All analyses were performed using SAS, release 9.2 (SAS institute Inc., 1999-2001, Cary, NC, USA). Statistical analyses, project management, clinical and site monitoring and quality control were performed by MediData (Modena, Italy) using internal procedures, in compliance with the Italian privacy law.

\section{RESULTS}

\section{Study population}

Two hundred and five RA patients meeting the inclusion/exclusion criteria were enrolled in the study. Mean duration of the disease (SD) was 14 years (7.2) and $80.5 \%$ of enrolled patients $(\mathrm{N}=165)$ were females. Mean age (SD) was 57.45 (12.07) years (ranging from 20.6 to 82.2 years). Patients were observed for up to 3.4 years from the start of rituximab treatment. Eligible patients had received at least one previous course of rituximab, and treatment was still ongoing at the time of recruitment. Twenty patients left the study during the 24-month follow-up period, 14 following a move from the area or unavailability, 3 due to a 
change of the clinical site or physician, 2 following withdrawal of informed consent; one patient died.

At the time of enrolment, 151 patients $(73.7 \%)$ presented with one or more concomitant diseases, most commonly cardiovascular diseases $(33.2 \%)$, endocrinological conditions $(19.5 \%)$ and other rheumatic diseases $(17.1 \%)$. Fifteen patients $(7.3 \%)$ had tuberculosis, none of which in the active phase.

At the start of the rituximab treatment, RF was available for 73 patients, 52 of which (71.2\%) were positive. Anti-CCP antibodies testing was performed in 50 patients at the start of the rituximab treatment, 34 of which $(68 \%)$ had positive results.

Fifty-seven percent of patients had received one anti-TNF $\alpha$ drug prior to the rituximab treatment, and $42.9 \%$ more than one TNF alpha inhibitor.

\section{Safety}

Throughout the observation period, extending from the start of the rituximab treatment to 24 months after the recruitment in the study, 82 patients (40\%) manifested one or more AE (Tab. I). Based on CTCAE criteria, AEs manifested following the start of the rituximab treatment were mild (grade 1 or 2) in $25.9 \%$ of patients, and moderate (grade 3 or 4 ) in $14.2 \%$.

Overall, out of $187 \mathrm{AE}$ reported, all of which grade 1 or 2 (CTCAE), the most common were infections (7\%), cystitis $(5.35 \%)$ and bronchitis (4.28\%). Eight cardiac events were reported (4.3\%), 4 of which were CTCAE grade 3 or 4 , and the remaining 4 were mild (grade 1 or 2). Twenty-six SAEs were observed from the start of the observation period (Tab. II).

Table I - Adverse events and serious adverse events.

\begin{tabular}{|l|c|}
\hline & $\begin{array}{c}\text { Total number } \\
\text { of evaluable } \\
\text { patients } \mathrm{n}=\mathbf{2 0 5}(\%)\end{array}$ \\
\hline Patients with adverse events & $82(40)$ \\
\hline $\begin{array}{l}\text { Patients with at least a grade 3 } \\
\text { or 4 adverse event }\end{array}$ & $29(14.2)$ \\
\hline $\begin{array}{l}\text { Patients with serious adverse } \\
\text { events }\end{array}$ & $23(11.2)$ \\
\hline
\end{tabular}

Table II - Classification of serious adverse events.

\begin{tabular}{|l|l|}
\hline & $\begin{array}{l}\text { Total number of } \\
\text { serious adverse } \\
\text { events } \mathbf{n = 2 6}(\%)\end{array}$ \\
\hline Death & $1(3.8)$ \\
\hline Life threatening event & $3(11.5)$ \\
\hline $\begin{array}{l}\text { Hospitalization or prolongation } \\
\text { of hospitalization }\end{array}$ & $17(65.4)$ \\
\hline $\begin{array}{l}\text { Persistent of significant } \\
\text { disability/incapacity }\end{array}$ & $2(7.7)$ \\
\hline Congenital anomaly/birth defect & $0(0)$ \\
\hline Important medical events & $9(34.6)$ \\
\hline
\end{tabular}

Multiple responses were possible for SAE classification.

Following the administration of a single course of rituximab and other concomitant therapies, including leflunomide $20 \mathrm{mg} /$ day p.o., and prednisone $25 \mathrm{mg} /$ day p.o, one patient died from septicemia.

In $42.8 \%$ of cases, AEs were deemed as being unrelated to administration of any drug, in $10.7 \%$ drug-correlation was seen as unlikely, in $18.2 \%$ possible and in $19.8 \%$ probable. In cases which were likely to be correlated with the pharmacological treatment, $64.6 \%$ of AEs were ascribed to rituximab administration, $40.4 \%$ to corticosteroid administration, and $37.4 \%$ to disease-modifying antirheumatic drugs.

Overall, $23 \%$ of AEs were infusion reactions; $70.6 \%$ of these were resolved: in $24.6 \%$ with no further intervention, and in $36.9 \%$ following pharmacological intervention.

Rituximab infusion was temporarily suspended in $10.2 \%$ of cases, and definitively discontinued in $6.4 \%$. The rate of infusion was reduced in $2.7 \%$ of cases.

\section{Rituximab treatment}

Sixty-eight (33.2\%) patients suspended treatment with rituximab.

Retreatment with rituximab was implemented in 192 patients (93.7\%), with 153 (79.7\%) undergoing at least 3 treatment cycles, $113(58.9 \%)$ at least 4 cycles, 69 $(35.9 \%)$ at least 5 cycles, $28(14.6 \%) 6$ cycles and $6(3.1 \%)$ reached 7 cycles. Seventy-five percent of patients started retreatment within 9.2 [95\% confidence interval (CI) 8.2-10.8] months from treatment initiation. 


\section{Response to rituximab}

An adequate response to the rituximab treatment $(\triangle \mathrm{DAS} 28>1.2)$ was observed in $50.8 \%$ (95\% CI: 39\%-63\%; $\mathrm{N}=63)$ and $63.2 \%$ (95\% CI: $51 \%-76 \%$; N=57) of patients at 12 and 24 months, respectively. Mean (SD) age of patients who did not respond to the rituximab treatment was 63 (11) years, while treatment responders were younger (56 years, $\mathrm{SD}=14$ ) ( $\mathrm{T}$ test $\mathrm{P}$-value $=0.04556$ ). The effect of the rituximab treatment appears to be significantly associated with initial disease activity. Indeed, at the start of treatment, responders displayed a higher mean DAS28 score than that observed in patients with a DAS28 score variation $\leq 1.2(5.45, \mathrm{SD}=1.20$ vs 4.50 $\mathrm{SD}=0.97$ ) (T-test $\mathrm{P}$-value $=0.0012$ ). With regard to time to response, the Kaplan-Meier analysis highlighted that approximately $50 \%$ of patients responded to the rituximab treatment within 7 (6.1-10) months.

Disease activity, clinical remission, quality of life and resource consumption

Median $25^{\text {th }}$ percentile- $75^{\text {th }}$ percentile $(25 \mathrm{p}$ $75 p)$ DAS28 score at the start of rituximab treatment was 5.1 (4.4-5.9), decreasing to 3.1 (2.3-4.1) after 24 months, thus indicating a low disease activity $(\mathrm{P}<0.0001$; Wil- coxon test of changes in DAS28 values). A decreasing trend was also observed for ESR and CRP values: median (25p-75p) ESR was 41 (20-67) at the start of rituximab, decreasing to 16 (9-30) at 24 months (Wilcoxon test $\mathrm{P}<0.0001)$; median $(25 \mathrm{p}$ $75 \mathrm{p}) \mathrm{CRP}$ at the start of treatment was equal to 1.7 (0.4-5.7), with a value of 0.6 (0.3-2.0) being achieved after 24 months (Wilcoxon test $\mathrm{P}=0.0014$ ).

After 6, 12 and 24 months, 27\%, 19\% and $26.3 \%$ of patients, respectively, achieved remission (DAS28<2.6) and 50.8\%, 54\% and $49.1 \%$ of patients displayed a moderate to good response (Fig. 1).

QOL was assessed by means of HAQ at 6,12 and 24 months in both patients in clinical remission and unremitted patients. Mean HAQ score was lower in patients in clinical remission than for those who were not in remission at each visit. After 6 months, a mean (SD) score of 0.63 (0.67) in patients in clinical remission, and $1.37(0.83)$ in those not in remission was seen $(\mathrm{P}<0.0002)$. After one year, a score of $0.61(0.47)$ was obtained for patients in remission, and $1.48(0.79)$ for unremitted patients $(\mathrm{P}<0.0001)$. After 24 months, subjects in remission displayed a mean (SD) HAQ score of 0.68 (0.68), while those not

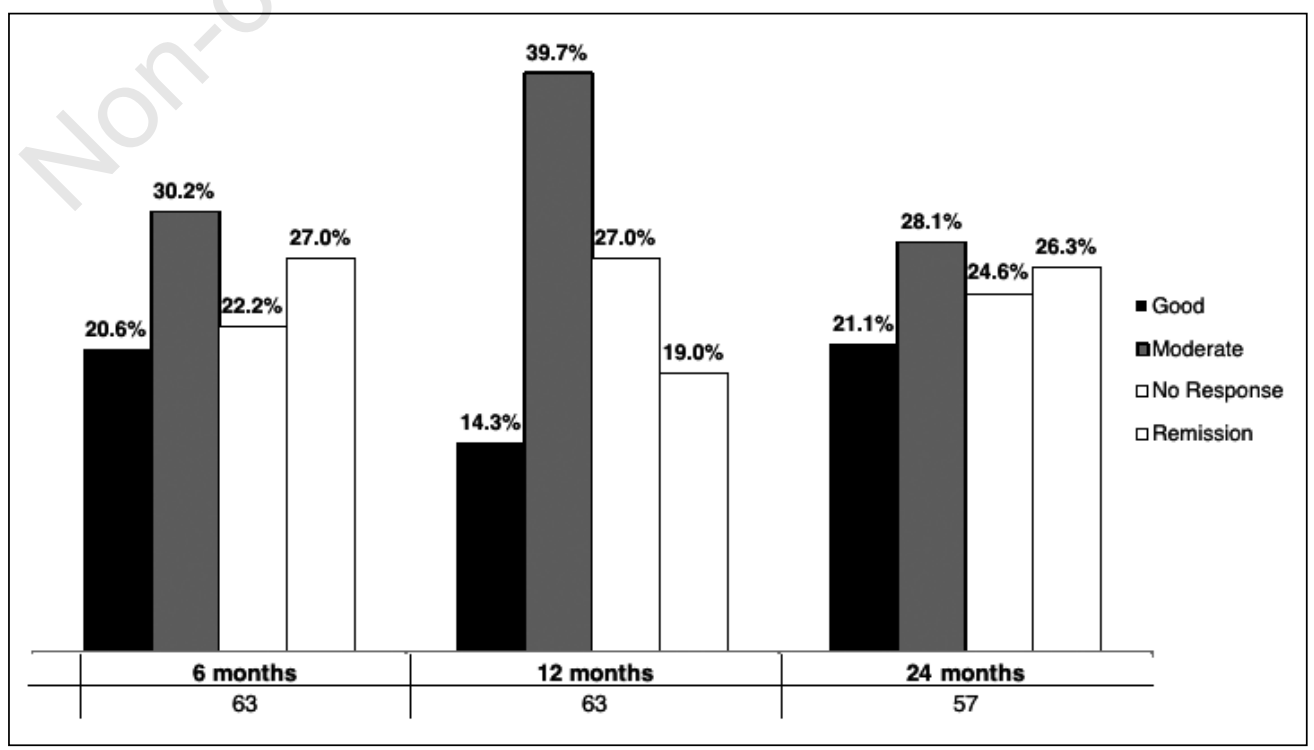

Figure 1 - Proportion of patients showing good, moderate, or no response according to the European League Against Rheumatism (EULAR) criteria and clinical remission (DAS28 <2.6) at 6,12 and 24 month follow-up visits. 
in remission showed a mean (SD) score of $1.57(0.83)(\mathrm{P}<0.0001)$.

During the observation period, $67 \%$ of patients $(\mathrm{N}=137)$ were not admitted to hospital, $8.8 \%(\mathrm{~N}=18)$ were hospitalized once and $11.2 \%(\mathrm{~N}=23)$ were hospitalized twice. Over the same period $87.3 \%$ of patients $(\mathrm{N}=179)$ made no visits to an emergency department, while 23 patients $(11.2 \%)$ visited it once, and $3(1.5 \%)$ twice.

Out of 205 enrolled patients, 128 (62.44\%) did not visit their general practitioner during the observation period, while 14 $(6.83 \%)$ did it approximately once/year.

\section{DISCUSSION}

The aim of this observational study was to assess the safety of rituximab over a period of observation lasting up to 3.4 years. Out of 205 patients enrolled in the study, 123 (60\%) failed to report the onset of any AEs, $53(25.9 \%)$ reported AEs of grade 1 or 2, while $29(14.2 \%)$ manifested at least one grade 3 or 4 AE. Overall, AEs were reported by 82 patients (40\%), a much lower percentage than that reported in the DANCER, REFLEX and RESET studies (85\% in all three studies after the first treatment cycle) $(10,11,26)$. However, the $40 \%$ observed in this study is higher than that reported in the CERERRA registry (from $13.9 \%$ in patients on rituximab monotherapy to $10.2 \%$ in patients receiving rituximab plus leflunomide) (27). This difference could be explained by the fact that patients from the CERERRA study were observed over a 12-month period, while the RUBINO study collected safety information for up to 3 years.

On the contrary, the percentage of patients manifesting SAEs was higher in the RUBINO (11.22\%) than in the DANCER and REFLEX studies. In the DANCER study, $7 \%$ of patients with SAEs were observed in the $2 \times 500 \mathrm{mg}$ rituximab group, while $2 \%$ of serious infectious events and $5 \%$ of non-infectious SAEs were observed in the $2 \times 1000 \mathrm{mg}$ rituximab group. In the REFLEX study, $7 \%$ of patients receiving rituximab plus methotrexate manifested SAEs. This difference could be explained by the comorbidity profile of enrolled patients and by the longer observation period of the RUBINO study compared to the other studies (up to 3.4 years vs 24 weeks in DANCER and REFLEX studies).

In the RUBINO study, the majority of AEs $(55.6 \%)$ were mild (grade 1) and in $42.8 \%$ of cases AEs were deemed unrelated to the administration of any drug, as suggested also by the type of events manifested.

Twenty-three percent of AEs were represented by infusion-related reactions, although definitive discontinuation of rituximab administration was required only in a few cases $(6.4 \%)$.

The changes in parameters and markers of RA during the observation period are of particular interest. Changes in DAS28 score appear to be related to initial DAS28 values. The DAS28 median score was 5.1 at baseline and subsequently showed a statistically significant decrease to 3.3 after 6 months, to 3.4 after 12 months and 3.1 after 24 months, indicating a satisfactory maintenance of the effect. Fifty percent of response to treatment was observed within 7 months. Over the 24-month study period, median ESR and CRP showed a significant decrease versus baseline. They dropped from 41 to 16 , and from 1.7 to 0.6 respectively and, as expected, mean HAQ score was significantly lower in patients in clinical remission after 6, 12 and 24 months of observation.

Based on the data available, gender and concomitant diseases did not appear to influence the response to rituximab. This study was conceived in 2008 , when the relationship between serological status and response to rituximab was still not fully understood. Indeed, this trial led to the observation that, in clinical practice, the choice of rituximab was based on the severity of the illness rather than on the serological status, and that rituximab was prescribed irrespective of the presence of concomitant diseases.

It is moreover noteworthy that no association was found between the rituximab treatment and the increased use of healthcare resources, as observed from the reduced number of visits to the GP and number of 
admissions to the emergency department.

This trial is however hampered by several limitations, the first of which is the absence of a control group. However, when the RUBINO study was conducted (2008-2009), Rituximab was the leading therapeutic option available in Italy for RA patients with a previous inadequate response or intolerance to TNF $\alpha$ inhibitors. For this reason, setting up a control group of patients treated with other drugs was impossible. A further limitation is represented by the retrospective phase of the trial, leading to difficulties in the collection of data, particularly as the collection of data in the RUBINO study started in 2009, when DAS28 evaluation was routinely performed less frequently than today. Accordingly, ESR and CRP were available at the start of the rituximab treatment for 93 and 94 subjects, respectively, out of a total of 205 recruited patients, and DAS28 was available at the start of the rituximab treatment for only 103 out of 205 enrolled patients.

In conclusion, the RUBINO study was the first trial to assess the safety of rituximab in routine Italian clinical practice in an uncontrolled population of patients with concomitant diseases. The results obtained confirm the satisfactory safety profile of rituximab in the treatment of RA patients in a real life setting, providing further support to data reported in previous studies.

\section{Acknowledgments}

The Authors acknowledge:

\section{The RUBINO study group}

The principal investigators of the RUBINO Study Group are as follows:

- Bombardieri Stefano, AOU Pisana, Ospedale Santa Chiara, Pisa;

- Lunardi Claudio, AOU Integrata, Verona;

- Adami Silvano, Centro Ospedaliero Valeggio sul Mincio, Valeggio sul Mincio (VE);

- Mathieu Alessandro, AOU Policlinico, Monserrato (CA);

- Pistone Giovanni, ARNAS Civico Di Cristina Benfratelli, Palermo;

- Sinigaglia Luigi, Istituto Ortopedico G. Pini, Milano;
- Concesi Claudia, Ospedale Guglielmo da Saliceto, Piacenza;

- Del Sante Giovanni, AOU di Parma, Parma;

- Di Giuseppe Paolo, Ospedale A. Perrino, Brindisi;

- Fusaro Enrico, AOU S. Giovanni Battista Ospedale Le Molinette, Torino;

- Laganà Bruno, AO Policlinico Sant'Andrea, Roma;

- Malavolta Nazzarena, Policlinico S. Orsola Malpighi, Bologna;

- Marasini Bianca, Istituto Clinico Humanitas, Rozzano (MI);

- Mazzone Antonino, Ospedale Civile di Legnano, Legnano (MI);

- Romeo Nicoletta, ASO S. Croce e Carle, Cuneo;

- Leardini Gianni, Ospedale SS Giovanni e Paolo, Venezia;

- Martin Martin Luis Severino, Ospedale Regina Apostolorum, Albano Laziale (RM);

- Migliore Alberto, Ospedale S. Pietro Fatebenefratelli, Roma.

\section{Sponsorship}

Roche Italia S.p.A.

MediData Studi e Ricerche (Modena) for Project Management, Statistical Analyses and data management:

- Alessandra Ori, clinical operations manager;

- Silvia Montanari, clinical research assistant;

- Silvia Gasparroni, Lucia Simoni, statisticians and medical writing support;

- Luca Zanoli, clinical data manager.

\section{REFERENCES}

1. Yelin E, Trupin L, Wong B, Rush S. The impact of functional status and change in functional status on mortality over 18 years among persons with rheumatoid arthritis. J Rheumatol. 2002; 29: 1851-7.

2. Brouwer WB, van Exel NJ, van de Berg B, Dinant HJ, Koopmanschap MA, van den Bos GA. Burden of caregiving: evidence of objective burden, subjective burden, and quality of life impacts on informal caregivers of patients with rheumatoid arthritis. Arthritis Rheum. 2004; 51: 570-7. 
3. Rupp I, Boshuizen HC, Jacobi CE, Dinant HJ, van den Bos GA. Impact of fatigue on healthrelated quality of life in rheumatoid arthritis. Arthritis Rheum. 2004; 51: 578-85.

4. Minaur NJ, Jacoby RK, Cosh JA, Taylor G, Rasker JJ. Outcome after 40 years with rheumatoid arthritis: a prospective study of function, disease activity and mortality. J Rheumatol. 2004; Suppl. 69: 3-8.

5. Pollard L, Choy EH, Scott DL. The consequences of rheumatoid arthritis: quality of life measures in the individual patient. Clin Exp Rheumatol. 2005; 5: 43S-52S.

6. Moreland L. Unmet needs in rheumatoid arthritis. Arthritis Res Ther. 2005; 7: 2S-8S.

7. Zink A, Listing J, Kary S, Ramlau P, Stoyanova-Scholz M, Babinsky K, et al. Treatment continuation in patients receiving biological agents or conventional DMARD therapy. Ann Rheum Dis. 2005; 64: 1274-9

8. Nikas SN, Voulgari PV, Alamanos Y, Papadopoulos CG, Venetsanopoulou AI, Georgiadis $\mathrm{AN}$, et al. Efficacy and safety of switching from infliximab to adalimumab: a comparative controlled study. Ann Rheum Dis. 2006; 65: 257-60.

9. Hjardem E, Østergaard M, Pødenphant J, Tarp U, Andersen LS, Bing J, et al. Do rheumatoid arthritis patients in clinical practice benefit from switching from infliximab to a second tumor necrosis factor alpha inhibitor? Ann Rheum Dis. 2007; 66: 1184-9.

10. Emery P, Fleischmann R, Filipowicz-Sosnowska A, Schechtman J, Szczepanski L, Kavanaugh $\mathrm{A}$, et al. The efficacy and safety of rituximab in patients with active rheumatoid arthritis despite methotrexate treatment: results of a phase IIB randomized, double-blind, placebo-controlled, dose-ranging trial. Arthritis Rheum. 2006; 54: 1390-400.

11. Cohen SB, Emery P, Greenwald MW, Dougados M, Furie RA, Genovese MC, et al. Rituximab for rheumatoid arthritis refractory to anti-tumor necrosis factor therapy: Results of a multicenter, randomized, double-blind, placebo-controlled, phase III trial evaluating primary efficacy and safety at twenty-four weeks. Arthritis Rheum. 2006; 54: 2793-806.

12. Popa C, Leandro MJ, Cambridge G, Edwards JC. Repeated B lymphocyte depletion with rituximab in rheumatoid arthritis over $7 \mathrm{yrs}$. Rheumatology (Oxford). 2007; 46: 626-30.

13. Keystone E, Fleischmann R, Emery P, Furst DE, van Vollenhoven R, Bathon J, et al. Safety and efficacy of additional courses of rituximab in patients with active rheumatoid arthritis: an open-label extension analysis. Arthritis Rheum. 2007; 56: 3896-908.

14. Keystone EC, Cohen SB, Emery P, Kremer JM, Dougados M, Loveless JE, et al. Multiple courses of rituximab produce sustained clinical and radiographic efficacy and safety in patients with rheumatoid arthritis and an inadequate response to 1 or more tumor necrosis factor inhibitors: 5-year data from the REFLEX study. J Rheumatol. 2012; 39: 2238-46.

15. Albers JMC, Kuper HH, van Riel PLCM, Prevoo MLL, Van't Hof MA, van Gestel AM, et al. Socio-economic consequences of rheumatoid arthritis in the first years of the disease. Rheumatology (Oxford). 1999; 38: 423-30.

16. Choi HK, Seeger JD, Kuntz KM. A cost-effectiveness analysis of treatment options for patients with methotrexate-resistant rheumatoid arthritis. Arthritis Rheum. 2000; 43: 2316-27.

17. Wong JB, Singh G, Kavanaugh A. Estimating the cost-effectiveness of 54 weeks of infliximab for rheumatoid arthritis. Am J Med. 2002; 113: 400-8.

18. Kobelt G, Lindgren P, Lindroth Y, Jacobson L, Eberhardt K. Modelling the effect of function and disease activity on costs and quality of life in rheumatoid arthritis. Rheumatology (Oxford). 2005; 44: 1169-75.

19. Benucci M, Iannazzo S, Sabadini L. Analisi di budget impact dell'utilizzo di rituximab nel trattamento dell'artrite reumatoide in Italia. Farmaeconomia e percorsi terapeutici. Farmeconomia. 2009; 10: 19-31.

20. Launois R, Payet S, Saidenberg Kermanac'h, Francesconi C, Riou França L, Boissier MC. Budget impact model of rituximab after failure of one or more TNF alpha inhibitor therapies in the treatment of rheumatoid arthritis. Joint Bone Spine Rev Rheum. 2008; 75: 688-95.

21. Arnett FC, Edworthy SM, Bloch DA, McShane DJ, Fries JF, Cooper NS, et al. The American Rheumatism Association 1987 revised criteria for the classification of rheumatoid arthritis. Arthritis Rheum. 1988; 31: 315-24.

22. Trotti A, Colevas AD, Setser A, Rusch V, Jaques D, Budach V, et al. CTCAE v3.0: development of a comprehensive grading system for the adverse effects of cancer treatment. Semin Radiat Oncol. 2003; 13: 176-81.

23. Fries JF, Spitz P, Kraines RG. Holman HR. Measurement of patient outcome in arthritis. Arthritis Rheum. 1980; 23: 137-45.

24. Felson DT, Anderson JJ, Boers M, Bombardier C, Chernoff M, Fried B, et al. The American College of Rheumatology preliminary core set of disease activity measures for rheumatoid arthritis clinical trials. The Committee on Outcome Measures in Rheumatoid Arthritis Clinical Trials. Arthritis Rheum. 1993; 36: 729-740.

25. Bruce B, Fries JF. The Stanford health assessment questionnaire. Dimensions and practical applications. Health Qual Life Outcomes. 2003; $1: 20$.

26. Haraoui B, Bokarewa M, Kallmeyer I, Bykerk VP, RESET Investigators. Safety and effectiveness of rituximab in patients with rheuma- 
toid arthritis following an inadequate response to 1 prior tumor necrosis factor inhibitor: the RESET Trial. J Rheumatol. 2011; 38: 254856.

27. Chatzidionysiou K, Lie E, Nasonov E, Lukina G, Hetland ML, Tarp U, et al. Effectiveness of disease-modifying antirheumatic drug cotherapy with methotrexate and leflunomide in rituximab-treated rheumatoid arthritis patients: results of a 1-year follow-up study from the CERERRA collaboration. Ann Rheum Dis. 2012; 71: 374-7. 